\title{
Aneurisma de artéria ilíaca em equinos
}

\author{
Iliac Artery Aneurysm in Equines
Ravy Guerra de Oliveira', Tairon Pannunzio Dias e Silva ${ }^{2}$ Ana Beatriz Nobrega do Santos' ${ }^{1}$ Carlos Henrique Camara Saquetti ${ }^{3}$, Samuel Rodrigues Alves ${ }^{4} \&$ Mariana Damazio Rajão ${ }^{4}$

\begin{abstract}
Background: Aneurysm is a dilation and a weakening of the vessel, usually 2 times higher than the normal diameter of the vessel. Considering the complexity of the definitive diagnosis of iliac artery aneurysm, it is necessary to use technology by imaging, aiming an early diagnosis. Thus, application of Doppler ultrasound allows the study of the horse's vascular system allowing various types of diagnoses among these members and vascularization for diagnosing thrombi in the abdominal aorta and mesenteric arteries in side verminotic aneurysm. The aim of this study was to describe the occurrence of two cases of aneurysm involving the aortic-iliac diagnosed by Doppler ultrasound.

Cases: The study describes two cases of the aneurism in horses diagnosed by Doppler ultrasound at the Veterinary Hospital of UPIS. The animals showed clinical signs of edema in the pelvic limb, with varied degree of lameness 3 to 4 , pain and wound did not heal and septic arthritis. The final diagnosis was obtained by Doppler ultrasound. In case one, Doppler ultrasound analysis indicated high arterial distension and blood flow disorganized, suggesting aneurysm. The patient did not respond adequately to therapy and due to the severity of these changes, the euthanasia was performed for definitive diagnostic. On the other hand, in case two, in the imaging examination, it was diagnosed with a dilated aortic Iliac left four times larger than the right and disorganized blood flow suggesting aneurysm. As a result of diagnosis and unfavorable prognosis, it was decided to stop sports activities by the animal mainly due to the progressive increase of the aneurysm.

Discussion: Between the two reported cases, one animal died with excessive extension of injury and in another it was possible to establish a palliative treatment being observed improvement in clinical symptomatology. The aneurysm was defined as a dilation of the artery, which may be congenital or acquired. In this case, it was not possible to define the main cause. However, their characteristics, visualized through ultrasound and necropsy corroborate to described in the literature, such as slow growth, achieve considerable volume may compress, displace and destroy neighboring structures. They also tend to increase in size until they break, causing bleeding. In the reports one and two it was found aneurysm verum, where in the animal of the report one was carried out euthanasia due to the rupture of the iliac artery. Surgical procedures are not usually indicated, due to the rapid evolution of the aneurysm culminating in hemorrhage and death of the patient. Ultrasound in these cases was also able to measure the extent of the lesion, to evaluate the blood flow and consequently the turbulence of blood. It is a disease with an unfavorable prognosis, by the progressive increase of the aneurysm or due to the possibility of aneurysm rupture. According to the results of this report can be concluded that Doppler ultrasound should be included in the routine practice of equine veterinary clinic, mainly in athletic horses to diagnose arterial lesions with complicated detection on clinical examination. The clinical symptomatology presented by the animals can be included in routine practice. The aneurysm of the iliac artery if was not diagnosed in time can cause death of the animal, however, if it was detected early, it can be established a palliative treatment extending the duration of animal life.
\end{abstract}

Keywords: aortic-iliac, disease, Doppler ultrasound.

Descritores: aortoilíaca, doença, ultrassonografia em modo Doppler. 


\section{INTRODUÇÃO}

Aneurisma pode ser definido como uma dilatação anormal e localizada de algum vaso sanguíneo. Contudo, têm sido descritos como maior importância clínica patológica quando afeta a aorta [2,14]. Na aorta várias lesões foram relatadas em equinos, tais como formação de aneurisma [8], aneurisma rompido [7], aneurisma por verminose [4] e aneurisma micótico [5].

A aorta descendente pode ser dividida em duas porções, torácica e abdominal. A aorta abdominal é um grande vaso ímpar que mede de 2 a $3 \mathrm{~cm}$ de comprimento e passa ventral a veia cava caudal para dentro da raiz mesentérica onde se subdividirá emitindo ramos colaterais principalmente para as paredes e cavidade abdominal, além da medula espinhal e da região pélvica.

Considerando a complexidade do diagnóstico definitivo do aneurisma arterial ilíaco, os vários tipos de lesões que podem ocorrer ao longo desse vaso assim como o curso que essa patologia se desenvolve, faz-se necessário o uso de tecnologia por imagem. Assim, a aplicação da ultrassonografia em modo Doppler, Segundo Cipone et al. [1], permite o estudo do sistema vascular do equino possibilitando vários tipos de diagnósticos entre esses a vascularização dos membros e para o diagnóstico de trombos na aorta abdominal e nas artérias mesentéricas secundários a aneurisma verminótico. É possível ainda, com a ultrassonografia em modo Doppler, avaliar as estruturas abdominais, realizar uma completa investigação da vascularização, determinar a velocidade e direção do fluxo sanguíneo, bem como identificar alterações vasculares [10]. Objetivou relatar a ocorrência de dois casos de aneurisma acometendo a aorta ilíaca diagnosticado pela ultrassonografia em modo Doppler.

\section{CASO 1}

Um equino fêmea da raça Puro Sangue Inglês (PSI), com aproximadamente nove anos de idade foi atendido no Hospital Veterinário da UPIS (HVT-UPIS) com um histórico clínico de dor severa no membro pélvico direito e claudicação. Após a anamnese, o animal foi submetido a uma avaliação física, sendo verificado claudicação de grau 4 de acordo com [11], aumento de volume no jarrete do membro pélvico direito que foi puncionado e encaminhada para o laboratório de bacteriologia da UPIS para realização na região da análise do liquido sinovial. Na palpação transretal verificou-se um aumento de volume próximo a bifurcação da artéria aorta ilíaca direita (Figura 1).

Como exames complementares foram realizados a cultura do líquido da região puncionada e ultrassonografia em modo Doppler (Ultrassom Veterinário Mindray Colorido - M5 VET). Na análise da cultura foi diagnosticado artrite séptica e as análises do ultrassom indicaram grande dilatação arterial e um fluxo sanguíneo desorganizado, sugerindo aneurisma.

Foi instituído um tratamento terapêutico à base de fenilbutazona (Equipalazone Injetável $\left.{ }^{\circledR}\right)^{1}$ na dose 4,4 $\mathrm{mg} / \mathrm{kg}$, SID, durante três dias, dimetilsufóxido (Dime$\left.\mathrm{sol}^{\circledR}\right)^{2}$ dose de $1 \mathrm{mg} / \mathrm{kg}$, SID, durante 2 dias, ceftiofur $\left(\text { Cef- } 50^{\circledR}\right)^{3}$ dose de $2,2 \mathrm{mg} / \mathrm{kg}$, BID, durante 5 dias, ducha de $15 \mathrm{~min}, \mathrm{SID}$, durante 5 dias no membro, 10 min de caminhada, SID, durante 5 dias e lavagem da articulação tarso metatársica com solução de ringer lactato $\left(\text { Baxter }^{\circledR}\right)^{4}$ seguida de infiltração com $250 \mathrm{mg}$ de sulfato de amicacina $\left(\operatorname{Novamin}^{\circledR}\right)^{5}$. O paciente não respondeu adequadamente à terapia e em decorrência da gravidade dessas alterações foi realizado a eutanásia para fins de diagnóstico definitivo.

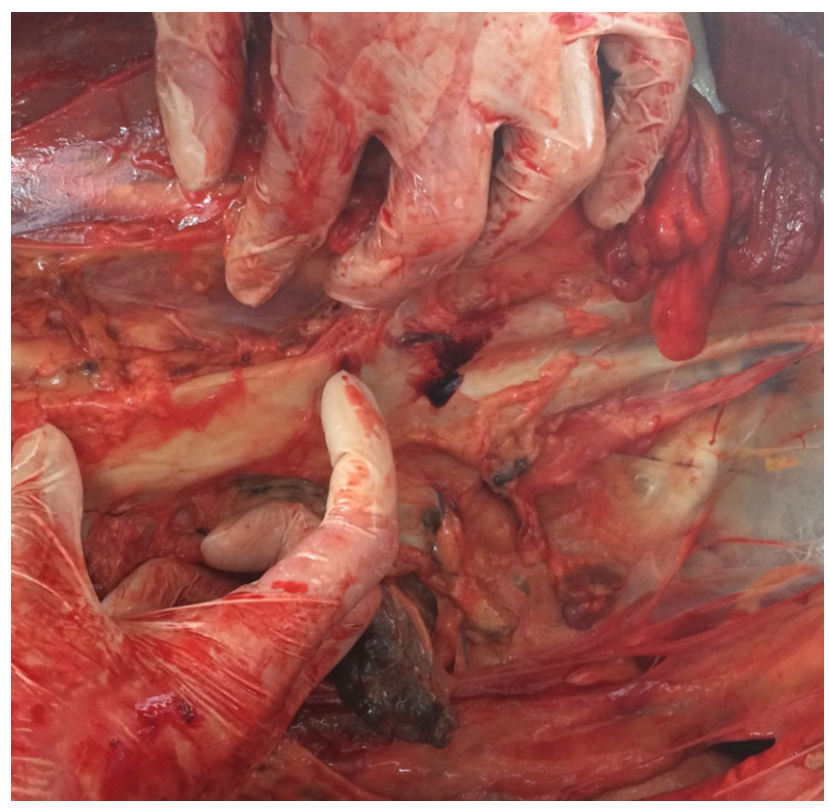

Figura 1. Extensão da lesão em decorrência do aneurisma na artéria ilíaca.

\section{CASO 2}

Uma égua da raça PSI, com 15 anos de idade, que foi encaminhado para o HVT- UPIS, com um edema no membro pélvico esquerdo e uma ferida localizado no metatarso do mesmo membro, com $8 \mathrm{~cm}$ de diâmetro, sem secreção, onde estava sendo tratada diariamente a aproximadamente 6 meses com limpeza 
da ferida com agua e sabão e uma pomada a base de neomicina, acetato de dexametasona, nistatina, sulfanilamida, oxido de zinco $\left(\text { Equiderm }^{\circledR}\right)^{1}$, sem apresentar avanço no processo de cicatrização.

No exame físico o animal apresentava claudicação grau 3 [11]. Com conhecimento de causa sobre o aneurisma de artéria ilíaca e sob essa suspeita clínica dessa patologia, foi solicitado, de modo semelhante ao caso 1 , a realização de exame ultrassonográfico em modo Doppler para visualizando da vascularização sanguínea da região. Ao exame de imagem foi diagnosticado uma dilatação da Aortoilíaca esquerda 4 vezes maior que a direita e um fluxo sanguíneo desorganizado (Figuras 2A e 2B) sugerindo aneurisma. Em decorrência do diagnóstico e do prognóstico desfavorável, optou-se por cessar as atividades esportivas pelo animal devido principalmente ao aumento progressivo do aneurisma.

Como tratamento paliativo, foi realizado ducha no membro afetado por 10 min no intuito de massagear
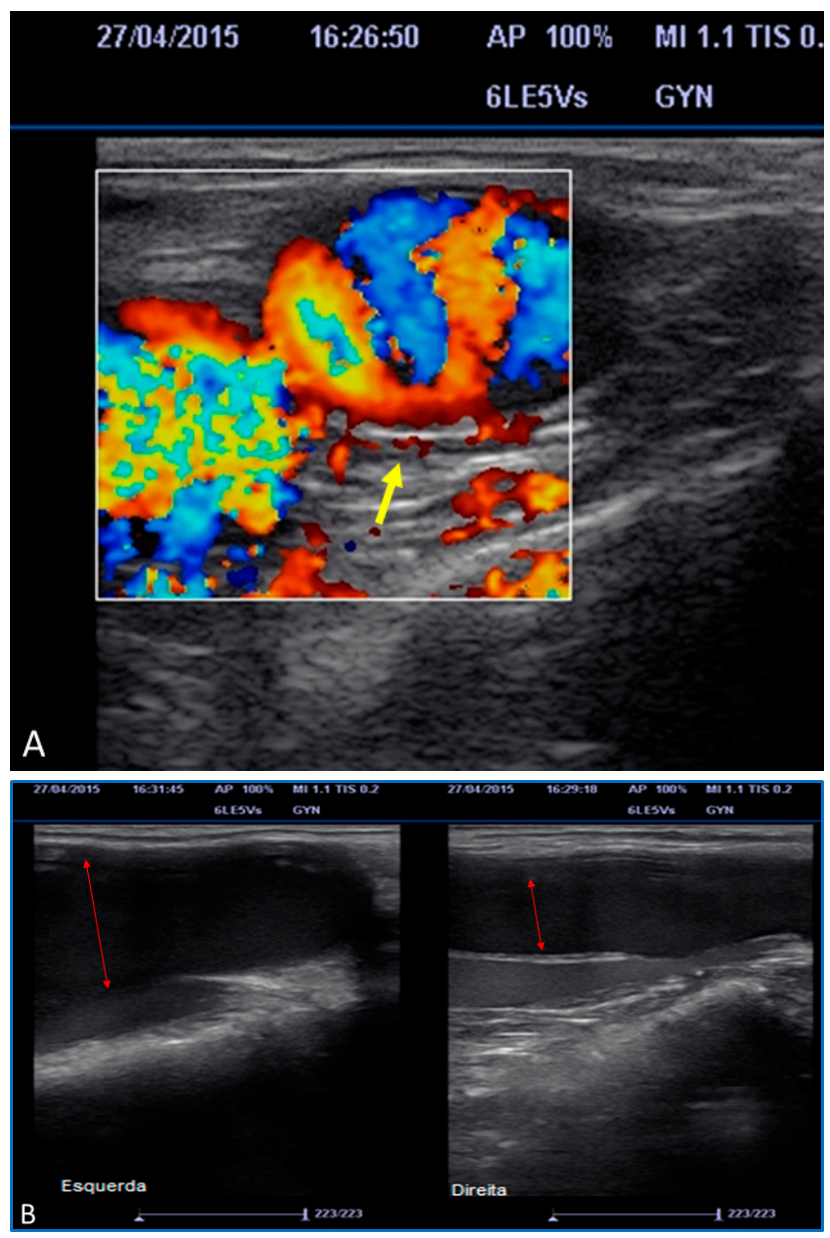

Figura 2. Ultrasonografia em modo Doppler. A- Fluxo sanguíneo desorganizado da ortoilíaca (seta). B- Diferença de tamanho da artéria, caracterizando aneurisma (seta). os tecidos, estimulando a circulação sanguínea e linfática e caminhadas leves de 20 min, durante 3 semanas, onde o animal apresentou melhora do seu quadro, com diminuição do edema.

\section{DISCUSSÃO}

Aneurismas da artéria ilíaca em equinos são raros. São mais comumente relatados quando afetam a aorta $[2,14]$ ou em lesões que afetam a aorta como além da formação [8], se relata também aneurisma rompido [7], aneurisma por verminose [4] e aneurisma micótico [5].

Para caracterização e diagnostico do aneurisma verdadeiro deve ter ocorrido uma dilatação maior que $150 \%$ do verdadeiro diâmetro da artéria com alterações (rompimentos ou alongamentos) em todas camadas da parede do vaso. No entanto, o diagnóstico é dificultado em regiões que a artéria apresenta locais de maior dilatação [9]. Em equinos essas alterações são raras e de grupo indefinido de lesões, consequentemente, poucas informações estão disponíveis quanto à sua ocorrência, classificação, apresentação e sinais clínicos, história natural e suas características quando avaliada no diagnóstico por imagem [6]. Os aneurismas têm sido diagnosticados com maior no post mortem devido a ruptura e morte súbita do animal. Sendo sua maior ocorrência na base da aorta [12].

Dessa forma, o aneurisma é definido como uma dilatação da artéria, podendo ser congênita ou adquirida. No presente caso não foi possível definir a causa principal. No entanto, suas características (Figuras 1 e 2), visualizadas através do ultrassom e da necropsia, corroboram com o descrito na literatura, como de crescimento lento, atingem volume considerável podendo comprimir, deslocar e destruir estruturas vizinhas. Além disso, tendem a aumentar de tamanho até se romper, provocando hemorragias.

Essa patologia possui três classificações [3]: aneurysma verum, aneurysma dissecans e aneurysma spurium. Essas categorias constituem diferentes patologias das doenças arteriais, sendo que o aneurisma dissecante e o pseudo são considerados falsos aneurismas, sendo incluídos na categoria de alterações saculares, fusiforme e micótica [13].

Nos casos 1 e 2 foi constatado aneurysma verum, onde o animal do caso 1 foi realizado a eutanásia em decorrência do rompimento da artéria ilíaca. Os procedimentos cirúrgicos normalmente não são indicados, devido a rápida evolução do aneurisma culminando em hemorragia e óbito do paciente. $\mathrm{O}$ 
aneurisma tem como consequência a alteração do fluxo sanguíneo (Figura 2A) e os sinais irão variar de acordo com o local acometido, como por exemplo claudicação, cólica, edema e sinais de insuficiência cardíaca congestiva, até a morte súbita.

A importância desse estudo se intensifica devido não somente à difícil classificação dentre os tipos de patologias mas também pela importância e necessidade cada vez mais iminente do uso de tecnologias do diagnóstico por imagem na medicina veterinária, onde a ultrassonografia em modo Doppler eficaz na formação do diagnóstico definitivo dos dois casos. A ultrassonografia, nesses casos, também foi capaz de mensurar a extensão da lesão, avaliar o fluxo sanguíneo e consequentemente o turbilhonamento do sangue (Figura 2A). O tratamento paliativo pode melhorar a qualidade de vida do animal. A única forma de prevenção do aneurisma é o controle parasitário. Trata-se de uma enfermidade com prognóstico reservado a desfavorável, pelo aumento progressivo do aneurisma ou devido a possibilidade de ruptura do aneurisma. Mesmo com os grandes avanços tecnológicos da medicina veterinária, a correção cirúrgica ainda não é uma opção.

Assim, em decorrência dessa tecnologia foi possível instituir tratamento paliativo (devido à complexidade do caso) no caso 2 que resultou em melhora, prolongando a vida do animal.
A sintomatologia clínica apresentada em ambos os casos foram semelhantes, onde os equídeos apresentaram dor aguda no membro pélvico, claudicação nível 3 e 4 de acordo com classificação de Stashak [11], aumento de volume a nível de jarrete assim como ferida em metatarso, podendo ser utilizado e incluídos na suspeita clínica de casos futuros que possam acontecer.

Os resultados do presente trabalho permitem concluir que a ultrassonografia em modo Doppler deve ser incluído na rotina prática da clínica veterinária de equídeos, principalmente em animais de exercício, para o diagnóstico lesões arteriais de difícil detecção ao exame clínico. A sintomatologia clínica apresentada pelos animais pode ser incluída na rotina prática. $\mathrm{O}$ aneurisma da artéria ilíaca, se não diagnosticado a tempo, cursa com a morte do animal, no entanto, se detectado possibilita a prescrição de um tratamento paliativo que prolonga a vida animal.

\author{
MANUFACTURERS \\ ${ }^{1}$ Hertape Saúde Animal S.A. Juatuba, MG, Brazil \\ ${ }^{2}$ Marcolab. Duque de Caxias, RJ, Brazil. \\ ${ }^{3}$ Agener União. Apucarana, PR, Brazil. \\ ${ }^{4}$ Baxter Hospitalar Ltda. São Paulo, SP, Brazil. \\ ${ }^{5}$ Teuto. Anápolis, GO, Brazil.
}

Declaration of interest. The authors report no conflicts of interest. The authors alone are responsible for the content and writing of the paper.

\section{REFERENCES}

1 Cipone M., Pietra M., Gandini G., Boari A., Guglielmini C. \& Venturoli M. 1997. Pulsed wave-doppler ultrasonographic evaluation of the common carotid artery in the resting horse: phisiologic data. Veterinary Radiology and Ultrasound. 38(3): 200-206.

2 Derksen F.J., Reed S.M. \& Hall C.C. 1981. Aneurysm of the aortic arch and bicarotid trunk in a horse. Journal of the American Veterinary Medical Association. (179): 692-694.

3 Goshal N.G. 1986. Coração e artéria do eqüino. In: Getty R. (Ed). Anatomia dos animais domésticos. Rio de Janeiro: Guanabara Koogan, pp.518-578.

4 Greatorex J.C. 1977. Diagnosis and treatment of 'verminous aneurysm' formation in the horse. Veterinary Record. (101): 184-187.

5 Okamoto M., Kamitani M., Tunoda N., Tagami M., Nagamine N., Kawata K., Itoh H., Kawasako K., Komine M., Akihara Y., Shimoyama Y., Miyasho T., Hirayama K., Kikuchi N. \& Tamiyama H. 2007. Mycotic aneurysm in the aortic arch of a horse associated with invasive aspergillosis. Veterinary Record. (160): 268-270.

6 Platt H. 1987. Vascular malformations and angiomatous lesions in horses: a review of 10 cases. Equine Veterinary Journal. (19): 500-504.

7 Roby K.A., Reef V.B., Shaw D.P. \& Sweeney C.R. 1986. Rupture of an aortic sinus aneurysm in a 15-year-old broodmare. Journal of the American Veterinary Medical Association. (189): 305-308.

8 Shirai W., Momotani E., Sato T., Kashima T. \& Itoi Y. 1999. Dissecting aortic aneurysm in a horse. Journal of Comparative Pathology. (210): 307-311.

9 Siablis D., Karnabatidis D. \& Katsanos K. 2004. Extracranial internal carotid artery aneurysm: report of ruptured case and review of the literature. Cardiovascular and Interventional Radiology. (27): 397-401. 
R.G. Oliveira, T.P.D. Silva, A.B.N. Santos, C.H.C. Saquetti, S.R. Alves \& M.D. Rajão. 2017. Aneurisma de artéria ilíaca em equinos. Acta Scientiae Veterinariae. 45(Suppl 1): 186.

10 Silva V.C., Mamprim M.J., Vulcano L.C. \& Doiche D.P. 2009. Ultra-sonografia Doppler e angiografia tomográfica computadorizada no diagnóstico de desvios portosistêmicos - revisão de literatura. Clínica Veterinária. 78: 70-78.

11 Stashak T.S. 1994. Claudicação em eqüinos equinos segundo Adams. 5.ed. São Paulo Rocca, pp.101-105.

12 Van D. Linde S.J.S., Kroneman J., Meulenaar H. \& Vos J.H. 1985. Necrosis and rupture of the aortic and pulmonary trunk in four horses. Veterinary Pathology. (22): 51-53.

13 Welling R.E., Taha A. \& Goel T. 1983. Extracranial carotid artery aneurysms. Surgery. 93(2): 319-323. 